\title{
Caracterización del estado nutricional y la calidad de vida en la micro cuenca del lago de Apanás, Jinotega, 2015
}

\section{Characterization of the nutritional status and quality of life in the micro watershed of Apanás Lake, Jinotega, 2015}

Evelyn Calvo Reyes ${ }^{1}$, Marcia Cordero Rizo ${ }^{1}$, Douglas José González Rivas², Jorge Isaac Soza Molinares²

${ }^{1}$ Docentes Investigadoras de la Facultad Multidisciplinaria UNAN-Matagalpa, ${ }^{2}$ Egresados de Ingeniería Agronómica

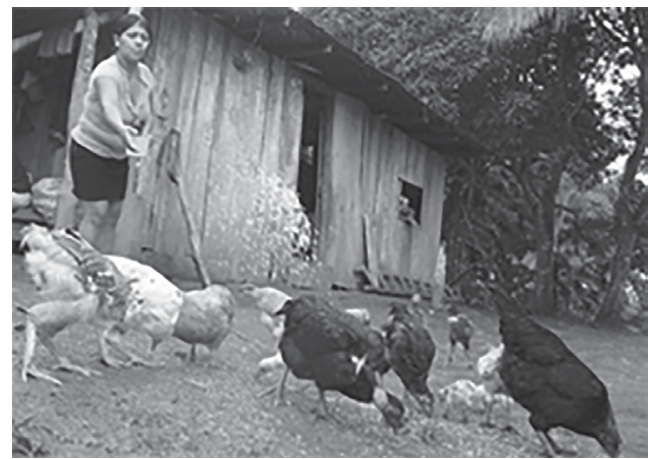

\section{RESUMEN}

El estudio se realizó en tres comunidades rurales del Municipio de Jinotega, Sisle, San Gabriel y Corinto Finca, todas pertenecientes a la micro cuenca del lago de Apanas, Jinotega, Nicaragua. El objetivo fue caracterizar el estado nutricional y la calidad de vida de las familias. La metodología utilizada fue bajo la perspectiva del enfoque cuantitativo, con componentes cualitativos. Se dispuso de una población de 253 familias y una muestra 72 familias, las cuales se tomaron de forma azarizada, para lo cual se utilizaron los criterios de: a) que en las familias existan un niños o niña menor de cinco años de edad, b) que la familia tuviera al menos seis años de vivir en las comunidades, c) que los jefes de familia estuvieran, anuentes a brindar la información necesaria. Para el análisis de la información se utilizó estadística descriptiva. Los resultados establecen que los niños o niñas menores de cinco años de edad nacen con buen peso, las madres cuidan del desarrollo del bebe mediante la asistencia del puesto de salud y el respectivo control prenatal por lo menos una vez al mes. Las madres proporcionan leche materna a los infantes de acuerdo a lo sugerido por el ministerio de salud de 3- 6 meses, lo que proporciona minerales y vitaminas necesarias para el crecimiento del bebe. Las familias poseen servicios básicos desde energía eléctrica, agua potable y saneamiento, además, poseen calidad de vida, viviendas en buen estado con paredes, piso y techos adecuados, que proporcionan seguridad, lo que evita el hacinamiento. Palabras clave: peso al nacer lactancia, servicios básicos.

\section{ABSTRACT}

The study was conducted in three rural communities in the municipality of Jinotega (Sisle, Finca San Gabriel and Corinto), all belonging to the micro basin of Lake Apanas, Jinotega, Nicaragua. The objective was to characterize the nutritional status and quality of life of the families. The methodology used was from perspective of quantitative approach with qualitative components. It had a population of 253 families and a sample of 72 families, which were taken at random using the following criteria, families with a child or girl under five years of age, that the family had at least six years living at the community, and householders willing to provide the information. For data analysis, descriptive statistics were used. The results establish that the boys and girls under five years of age are born with good weight, mothers take care for the development of the baby by attending the health center and the respective prenatal care at least once a month. Mothers provide breast milk to infants during a period of 3- 6 months, according to suggestion by the health ministry, which provided minerals and vitamins needed for growth of the baby. Families have basic services such as electricity, drinking water and sanitation. Furthermore, families have quality of life, housing in good condition with adequate walls, floor and ceilings, which provide security and preventing overcrowding.

Recibido: 1 de marzo 2016

Aceptado: 10 de mayo 2016 


\section{MATERIALES Y MÉTODOS}

El estudio se llevó acabo en la micro cuenca del Lago de Apanás ubicado a seis $\mathrm{km}$ de la ciudad de Jinotega en las comunidades de San Gabriel, Sisle y Corinto Finca, la cual posee una extensión de $54.15 \mathrm{~km}^{2}$. El clima es templado, con altitud de $955 \mathrm{msnm}$. La microcuenca se localiza entre las coordenadas de 13010 " 42 " de latitud Norte y 85058"31" longitud Oeste, con temperatura que oscila entre $23-27 \mathrm{C}^{\circ}$ (INETER, 2003).

Descripción de la recolección de los datos. El estudio se desarrolló en marzo -Junio del 2015. Los datos fueron recopilados en la época seca, entre los meses de marzo - Mayo. El estudio es de naturaleza transversal, no experimental con enfoque mixto con variables cualitativas y cuantitativas. Los datos se procesaron en Excel, mediante estadística descriptiva. La recopilación de información secundaria se realizó mediante el instrumento de la encuesta, a través de un listado proporcionado por el Consejo Sandinista de Desarrollo Humano (CSDH)

Para caracterizar el estado nutricional de las familias se utilizaron indicadores del historial clínico de madre, tales como: edad del embarazo, visita al control prenatal duración de la lactancia, y enfermedades presentadas en el infante. Por otro lado, se consideraron las condiciones sociales existentes en las familias como son: escolaridad, calidad de la vivienda servicios básicos y saneamiento

La población estuvo constituida por 253 familia y la muestra se calculó a partir de la fórmula propuesta por Schenaffer, Richar \&Mendenhall (1987), lo que proporciono una muestra de 72 familias, distribuida en Sisle 36, San Gabriel 19 y Corinto finca 17. La técnica de recolección de la información fue la encuesta y la observación mediante el pesaje de los niños y revisión de las tarjetas de control.

\section{RESULTADOS Y DISCUSIÓN}

Edad promedio de mujeres en estado de embarazo en las comunidades de Corinto Finca, San Gabriel y Sisle. Los resultados del estudio muestran que la mayoría en las mujeres en estados de embarazo engendra con edades menores de 20 años. Los promedios obtenidos fueron, $90 \%$ en Corinto finca, $85 \%$ en San Gabriel y $80 \%$ en Sisle. El resto de mujeres, en las tres comunidades, engendra a edades superiores a los 30 años (10 por ciento en Sisle, $15 \%$ en San Gabriel y $10 \%$ en Corinto finca. Según la atención integrada a las enfermedades prevalentes de la infancia (MINSA, 2009) en los cuadros de procedimientos de evaluación, clasificación y el trato de la embarazada durante el parto, determina que la edad es un factor muy importante y para no poner en peligro al bebe considera que la edad debe estar entre los 20-35 años de edad, clasificado como un parto de bajo riesgo.
La figura 1 representa la frecuencia con que la mujeres en estado de embarazo realizan visitas el puesto de salud para el debido control tanto de su salud como la del bebe, según el MINSA (2009) establece que por lo mínimo la madre debe de realizarse el control prenatal $1 \mathrm{vez}$ al mes, podemos observar que en la mayoría las madres asisten al puesto de salud mensualmente, para el debido control prenatal lo cual indica que las madres vigilan el desarrollo de sus hijos.

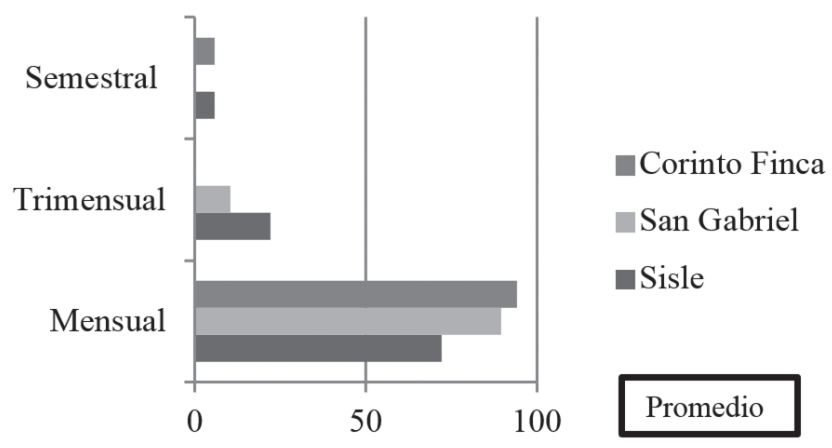

Figura 1. Frecuencia de control prenatal al puesto de salud de las mujeres en estado de embarazo de las comunidades Corinto finca, san Gabriel, y Sisle 2015. Fuente Resultado de Investigación.

La figura 2 represente el peso promedio de los niños al nacer Según la Atención Integrada a las enfermedades prevalentes de la Infancia MINSA (2009), establecen que el recién nacido de alto riesgo los siguientes signos como: peso al nacer $<2000 \mathrm{~g} \mathrm{o}>4000$ gr , semanas de gestación $<35$ Temperatura $>35.5 \mathrm{C}^{\circ}$ y otras anomalías de salud que presente la madre y mediano riesgo peso al nacer entre $2000 \mathrm{y}$ $2500 \mathrm{~g}$ edad gestacional entre 35-37 semanas y en bajo riesgo un recién nacido vigoroso, Rosado ,llanto fuerte, respiración normal, activo peso $>2500 \mathrm{~g} \mathrm{y}<4000 \mathrm{~g}$ con edad gestacional $>37<42$ semanas y según los resultados podemos observar que los niños posee un buen peso al nacer mayor o entre los 2500 gramos y en menor escala los niños al nacer obtienen un peso mayor de lo recomendado por la AIEPI,2009).

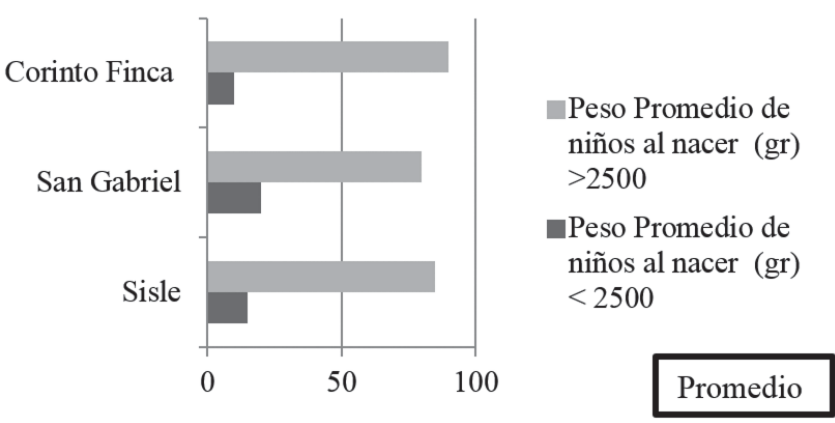

Figura 2. Peso promedio de los niños al nacer las comunidades Corinto finca, San Gabriel, y Sisle 2015. Fuente: Resultados de Investigación. 
La figura 4 representa la duración de la lactancia materna en los niños en las comunidades en estudio se considera en tiempo buena ya que las madres amamantan mayor de 2 meses hasta los doce meses y muy pocas amamanta más de los 24 meses, de acuerdo con el informe de todas las regiones del mundo están experimentando cambios en la prevalencia de desnutrición crónica .En el caso de América Latina la prevalencia bajo del 22\% entre 1990-2008, También hay noticias alentadoras para el caso de la lactancia materna exclusiva como es el caso de Colombia y Perú en la región donde prevalencia de LME aumento en un 30\%; de $11 \%$ a $47 \%$ en el caso de Colombia entre (1995-2005) y de un 33\% a $64 \%$ para el caso de Perú (entre 1992-2007). La iniciación temprana de la lactancia materna se encuentra en un $48 \%$ (todas las regiones del mundo excluyendo China.

MINSA (2009) indica que la promoción de la lactancia materna en atención primaria de salud, y lactancia materna es el corazón de la seguridad alimentaria de la economía de los recursos naturales, sostenibles de las comunidades del medio ambiente y del futuro de las personas y nuestro planeta

El Ministerio de Salud de Nicaragua, promueve la lactancia materna, desde la primera media hora después del parto y continuar la lactancia exclusiva hasta los seis meses ya que reúne todos los requerimientos nutricionales con excepción del a vitamina $\mathrm{D}$ y Hierro en los lactantes prematuros y con bajo peso del nacimiento y combinarla con alimentación complementaria hasta los dos años (OMS, 2003)

La lactancia materna a aumentado en 3\% (19932007) de 29 a $31 \%$ (ENDESA, 2007) la misma encuesta evidencia que un $94 \%$ de los niños nacidos en el 2007; al menos una vez recibieron lactancia.

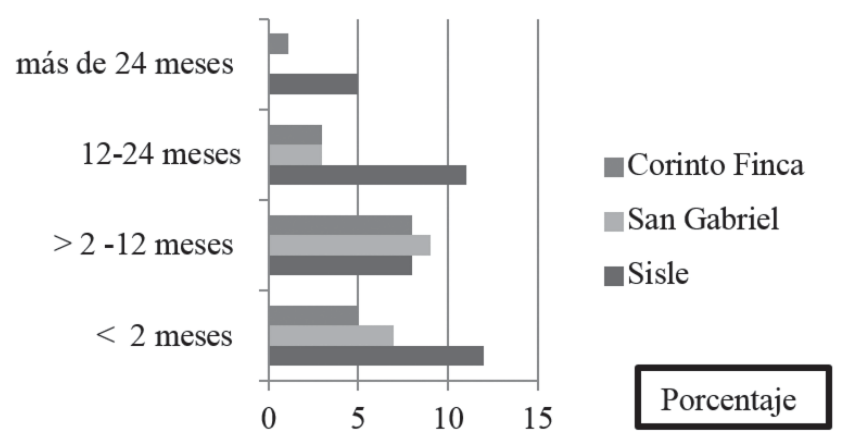

Figura 3. Promedio de la duración de la lactancia (meses) en los niños las comunidades Corinto finca, San Gabriel, y Sisle 2015. Fuente: Resultado de Investigación.

La figura 4 indica las enfermedades más comunes en el infante se ven afectados por las vías respiratorias entre ellas gripe, tos, asma, bronquitis y neumonía en mayor porcentaje son afectadas los de la comunidad de Sisle los niños.

Los principales resultados del estudio en un total de 110 programas y 10 planes y políticas en 8 países partici- pantes del estudio, en donde se identificaron los principales problemas nutricionales y la población objetivo, lo cual se refleja en los siguientes datos desnutrición Crónica (51\%) desnutrición global (45\%) anemia (37\%) Desnutrición aguda (36\%) Sobre peso y obesidad (36\%) Deficiencia de Vit A (25\%) Bajo peso al Nacer (26\%) Deficiencia de Folatos (15\%) Deficiencia de Zinc (14\%), se logró determinar criterio para la focalización de los programas los cuales son ubicación geográfica (72\%) grupos etarios (63\% estado VIH positivo (20\%) Factores biológicos (41\%) pobreza extrema (39\%) pobreza (28\%) y otros (5\%) étnico-cultural (3\%), lo cual recomienda compromisos políticos gubernamentales para la erradicación de la desnutrición infantil y materna dentro de las agendas nacionales y regionales.

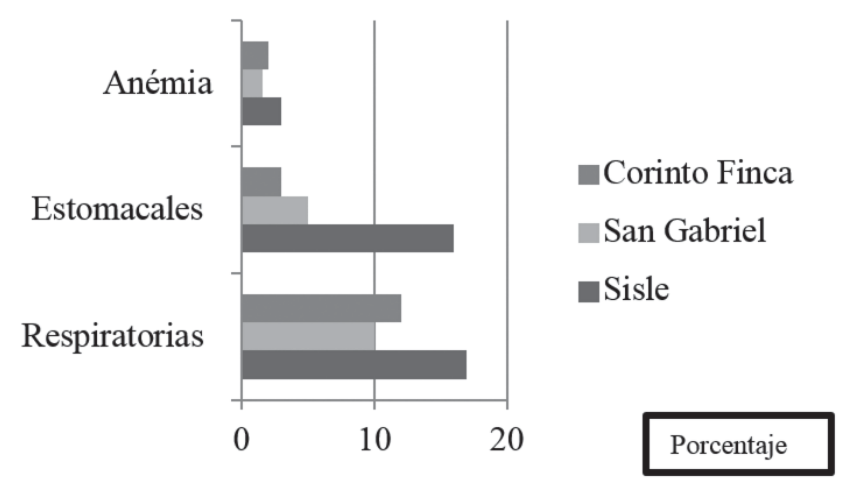

Figura 4. Enfermedades más comunes en los infantes en los niños de las comunidades Corinto finca, San Gabriel, y Sisle 2015. Fuente: Resultados de Investigación.

El tipo de familia existente en las comunidades en estudio, prevalece el tipo de familia extendida en Sisle el (77.78\%), San Gabriel (58.33\%), y Corinto Finca (80.56\%), donde se comparte con por lo menos uno o más persona o familiar, paterno o materno.

Luengo y Aroman (2000), manifiesta que una estructura familiar es la relación que existe entre los miembros que habitan en el hogar, realizando cada uno funciones diferentes para satisfacer sus necesidades.

Con el estudio de Toruño, 2014 se encontró que la mayoría de las familias en la trinidad son de estructura Nuclear, donde solo habita en los hogares padres e hijo lo cual contribuye a una mejor estabilidad en el hogar.

El promedio de integrantes de las familias en las comunidades en estudio, donde prevalece que en cada hogar existe un promedio de 1- 5 integrantes lo cual coincide con Avendaño (2009), expresa que en las zonas urbanas y rurales establece que el tamaño de las familias es un promedio 5- 6 integrantes; y lo encontrado con la información de Obando (2010) en tres comunidades del municipio de Tisma en el Departamento de Masaya existe un promedio de 1-6 integrantes ,donde existe hacinamiento en los hogares.

El nivel de escolaridad de los jefes de familias en las comunidades en estudio, CAAEC (2007) expresa que el ni- 
vel educativo es considerado un factor clave en el nivel de la vida familiar y como forma de combatir la pobreza, donde predomina que los jefes de familias no saben leer ni escribir, leer y escribir en $87 \%$ en las comunidades en estudio y 13 $\%$ inicio la secundaria, este indicador es de gran importancia tanto económicamente y social, permitirá mejor comunicación; cuyos resultados son similares obtenidos por Balmaceda y Fragas 2014) en la micro cuenca de Moyúa del municipio de Ciudad Darío, donde prevalece el nivel de escolaridad de primaria. La disponibilidad de la educación en la zona es limitadas solo cuenta con dos secciones y los maestro en su mayoría son empíricos.

Según PNUD y OIT (2009), señala que en Nicaragua los principales problemas de vivienda son los materiales con que están construidas generalmente plástico, zin, madera y no satisfacen las necesidades de los servicios básicos necesarios de las familias.

Una vivienda para considerarse como digna debe de cubrir de forma satisfactoria las necesidades básicas sanitarias como: privacidad, comodidad, funcionalidad, ubicación, seguridad en la tenencia y provisión de agua potable y medios de eliminación de excretas y basura (agua de drenajes, alcantarillados) para prevenir enfermedades que alteran el estado de la salud de las personas.

Material de las paredes de las viviendas de las familias en estudio prevalece las paredes de concretos y de madera en menor escala de plásticos con techos de Zinc en menor escala la teja rustica lo cual argumentan los encuestado que han tenido el apoyo de gobierno para mejorar sus vivienda.

Cuyos resultados coinciden con Balmaceda y Fargas, (2014) donde los materiales de las viviendas en la micro cuneca de Moyúa es de concreto, madera, techo de zinc y piso de tierra, en las comunidades en estudio posee piso en Sisles $47 \%$, es de tierra, $3.78 \%$ embaldosados, en San Gabriel $13.66 \%$ Piso de Tierra, $5.56 \%$ embaldosado y $5 \%$ de cerámica en la comunidad de corinto finca $18.06 \%$ Piso de Tierra , $6.94 \%$ embaldosado lo que indica que las familias poseen en su mayoría condiciones necesarias para la protección de las familias de enfermedades a los menores.
En lo que corresponde en el servicio de saneamiento en un $92 \%$ poseen letrinas y en un $8 \%$ todavía no posee servicios sanitarios, lo cual indica que las familias, se abastecen del vital líquido en un $85 \%$ poseen agua potable y en $15 \%$ se abastecen de las fuentes de rio y quebrada, todas las comunidades poseen servicios de energía eléctrica en su mayoría.

El estado de la vivienda en las comunidades en estudio en lo que prevalecen el buen estado y regular debido a la presencia de la contribución del gobierno con el programa de plan techo, lo planteado por Rojas (2009) Plantea que una vivienda digna debe de satisfacer simultáneamente los requisitos a) que la vivienda este ocupada por una familia b) que no tenga más de dos ocupantes por cuarto habitable en el medio urbano y no más de 2.5 en el rural c) que no esté deteriorada d) que cuente con los servicios de básicos necesarios.

\section{CONCLUSIONES}

Los resultados permiten concluir que las madres en las comunidades en estudio atienden a las recomendaciones del Ministerio de Salud (puesto de salud ) ya que la mayoría de los niños menores de cinco años de edad, nacen con buen peso, las madres frecuenta el puesto de salud para su respectivo control por lo menos una vez al mes y el proporcionan lactancia materna como mínimo 6 meses lo cual es propuesto por Atención Integrada a las enfermedades prevalentes de la infancia. A pesar de la distancia del puesto de salud, las madres deben de viajar en algunos casos hasta San Rafael del Norte.

Las familias poseen calidad de vida según los indicadores de estructura familiar, calidad de la vivienda, infraestructura el porcentaje de hacinamiento es bajo de $2 \%$, poseen los servicios básicos de saneamiento, letrina, agua, y energía eléctrica, el nivel de escolaridad de los jefes de familia es bajo lo cual podría estar incidiendo en los ingresos de la economía que podrían aportar a la familiar, existiendo escuela con los niveles de primaria completa y multigrado ,están organizados en los comité Sandinista de desarrollo Humano ,lo cuales están en constante vigilancia social y salud en sus respectivas comunidades

\section{REFERENCIAS BIBLIOGRÁFICAS}

Avendaño 2009. La pobreza en Nicaragua. Disponible en www. Wordpress.com

Balmaceda; Fragas. 2014. Caracterización agrosocioeconomica de las unidades de producción en la micro cuenca de Moyua, Ciudad Darío, Matagalpa segundo semestre, 2013. Tesis para optar al título de ingeniero agrónomo de la Facultad Multidisciplinaria Matagalpa.

INETER (Instituto Nicaragüense de Estudios Territoriales). 2003. Datos climatológicos de Jinotega, Apanas, Nicaragua.

Luengo; Aroman. 2000, Estructura familiar y satisfacción parental México DF, 1 edición

MINSA (Ministerio de Salud, NI). Atención Integrada a las enfermedades prevalentes de la infancia.

Obando, AJ. 2010. Seguridad alimentaria y nutricional de las familias rurales de las comarcas: Los 24, Las cortezas, y Las Montañas $\mathrm{N}^{\circ} 2$ del departamento de Masaya, municipio de Tisma, noviembre 2009.

Rojas, J. 2009. Cooperativismo y Desarrollo Humano: una propuesta para la medición "Tesis Doctoral del Colegio de Posgraduados Puebla, México para optar al título de Doctor en Desarrollo Rural 